УДК 551.24.02:528.344

ИССЛЕДОВАНИЕ ВЛИЯНИЯ СЛОИСТОСТИ И СФЕРИЧНОСТИ ЗЕМЛИ НА РАСЧЁТ КОСЕЙСМИЧЕСКИХ СМЕЩЕНИЙ И РЕЗУЛЬТАТЫ ИХ ИНВЕРСИИ

1,2Нечаев Г.В., ${ }^{1,2}$ Шестаков Н.В., ${ }^{3}$ Такахаши Х.

${ }^{\prime}$ ФГАОУ ВО «Дальневосточный федеральный университет», Владивосток, ${ }^{2}$ ФГБУН Институт прикладной математики Дальневосточного отделения Российской академии наук, Владивосток, e-mail: shestakov.nv@dvfu.ru; ${ }^{3}$ Институт сейсмологии и вулканологии Хоккайдского университета,

Cannopo, e-mail: hiroaki@sci.hokudai.ac.jp

Точность моделирования деформаций земной коры, вызванных землетрясениями, зависит не только от детальности модели очага сейсмического события, но и от сложности и структуры используемой модели Земли. Соответственно, при решении обратной задачи (определение параметров землетрясения в результате инверсии косейсмических смещений) используемая модель внутреннего строения Земли также оказывает влияние на конечный результат. В системах предупреждения о цунами, основанных на применении методов Глобальных навигационных спутниковых систем (ГНСС), одна из главных задач - определить, насколько возможно точное определение параметров землетрясения, от которых зависит надёжность последующего моделирования цунами. В данной работе рассмотрено влияние модели Земли на величины косейсмических смещений при прямом их моделировании. Рассмотрены эффекты, вызываемые влиянием слоистости, сферичности и обоих факторов одновременно. При моделировании использовались три гипотетических очага с магнитудами $\mathrm{M}_{w} 7,5 ; 8,0$ и 8,5, принадлежащих Камчатской зоне субдукции. В результате моделирования определены вертикальные поля смещений морского дна и Камчатского полуострова, а также горизонтальные смещения на пунктах региональных ГНСС-сетей. Сравнение полученных смещений говорит о том, что учёт слоистости и сферичности приводит к уменьшению значений расчётных подвижек, кроме того, влияние на их величины оказывает и дальность расположения ГНСС-станций от очага землетрясения, а также магнитуда события. Рассчитанные наборы смещений на пунктах ГНСС-сетей далее были инвертированы по методу Мацууры и Хасегавы, в котором модель Земли представлена однородным полупространством. При инверсии определялась только величина смещения в очаге и магнитуда, остальные параметры считались известными. Сравнение полей вертикальных смещений морского дна, соответствующих инвертированным очагам, с опорными значениями показало, что пренебрежение слоистостью и сферичностью Земли в работе СПЦ выражается в некоторой недооценке цунамиопасности.

Ключевые слова: ГНСС-методы, предупреждение о цунами, инверсия, косейсмические смещения

\title{
INVESTIGATION OF IMPACT OF THE EARTH MODEL TO COSEISMIC DISPLACEMENTS CALCULATION AND RESULTS OF THEIR INVERSION
}

\author{
${ }^{1,2}$ Nechaev G.V., ${ }^{1,2}$ Shestakov N.V., ${ }^{3}$ Takakhashi Kh.
}

${ }^{1}$ Far Eastern Federal University, Vadivostok;

${ }_{2}^{2}$ Institute for Applied Mathematics of Far Eastern branch of Russian Academy of Science,

Vladivostok,e-mail: shestakov.nv@dvfu.ru;

${ }_{3}^{3}$ Institute of Seismology and Volcanology of Hokkaido University, Sapporo, e-mail: hiroaki@sci.hokudai.ac.jp

Accuracy of modeling of Earth's crust deformation caused by earthquakes depends not only on seismic source model details but also on particularity of utilized Earth's model. Respectively for the inverse problem (determination of earthquake's parameters during the inversion of coseismic displacements) used Earth's model affects the result too. One of the main task of tsunami warning systems (TWS), which are based on Global Navigation Satellite Systems (GNSS) is possibly accurate determination of earthquake's parameters that strongly affect the reliability of tsunami modelling. Influence of Earth's model to coseismic displacements' values obtained by direct modelling is reviewed in the paper. Effects of stratification and spherecity were considering individually and together. Three hypothetical sources of magnitudes $M_{w} 7,5,8,0$ and 8,5 belonging to Kamchatka subduction zone were taken to modelling. Fields of vertical coseismic displacements of sea bottom and Kamchatka peninsula and horizontal offsets at the locations of local GNSSnetworks' stations were calculated. Comparison of obtained displacements shows that considering of stratification and sphericity leads to reduction of modelled offsets. In addition, distance of GNSS-stations from earthquake source and its magnitude affect the coseismic displacements too. Calculated sets of displacements at the GNSS-networks' points were inverted by Matsuura \& Hasegawa method that utilize homogeneous half-space Earth's model. The only displacement in the source value and magnitude were determined by the inversion. Comparison of coseismic displacements' vertical fields corresponding to inverted sources with reference ones showed some underestimation of tsunami hazard due to neglecting of stratification and sphericity by TWS.

Keywords: GNSS-methods, tsunami warning, inversion, coseismic displacements

В последние годы активно развиваются системы предупреждения о цунами (СПЦ), основанные на использовании методов Глобальных навигационных спутниковых систем (ГНСС). В отличие от СПЦ, использующих сейсмические методы, системы нового типа нацелены на определение параметров очага землетрясения и, далее, оценку 
начальных движений морского дна и характеристик цунами. Подобный принцип используется в системах REGARD (Япония) и G-larmS и G-FAST (США) [1-3]. Схема работы этих систем схожа:

1) детектирование косейсмических смещений, порождённых землетрясением, ГНССметодами (либо их комбинированием, например, с обработкой сейсмологических данных);

2) определение параметров землетрясения путём решения обратной задачи (инверсия косейсмических смещений);

3) моделирование смещений морского дна с использованием полученных параметров землетрясения;

4) расчёт характеристик цунами, заплесков волн на побережье и определение зоны затопления.

От системы требуется не только точность оценки цунамиопасности, но и оперативность её получения. Одним из факторов, влияющих на точность оценки параметров землетрясения и смещений морского дна, является используемая системой модель внутреннего строения и физических свойств Земли. Более подробные модели, участвующие в инверсии и моделировании смещений морского дна, теоретически должны способствовать более точной оценке параметров очага и цунами. Однако использование сложных моделей неизбежно приведёт к дополнительным временным затратам при расчётах и негативно отразится на быстродействии системы. Следовательно, необходимо соблюсти разумное соотношение скорости обработки данных и точности получаемых результатов. Даже в наиболее совершенной на данный момент СПЦ REGARD [1] модель Земли представлена однородным полупространством (инверсия происходит по методу Мацууры и Хасегавы [4]), т.е. не учитывает ни слоистости земной коры, ни сферичности её поверхности.

В работе [5] уже рассматривалось влияние слоистости и сферичности на величины горизонтальных косейсмических смещений, смоделированных с использованием параметров Суматра-Андаманского землетрясения $\mathrm{M}_{\mathrm{w}}$ 9,2. В отличие от [5], в настоящей работе исследуется необходимость учёта слоистости и сферичности Земли в работе СПЦ, поскольку одним из ключевых аспектов её работы является оперативное моделирование косейсмических движений морского дна. Зависимость быстродействия гипотетической системы от задействованных моделей Земли рассматриваться не будет, поскольку здесь большую роль играют вычислительные возможности центра обработки системы.

\section{Расчёт косейсмических смещений}

Наиболее цунамиопасными регионами России являются Камчатка и Курильские острова, и наличие здесь СПЦ, основанной на ГНСС-методах, крайне желательно [6]. Для простоты рассмотрены только гипотетические землетрясения: три очага, с моментными магнитудами $\mathrm{M}_{\mathrm{w}} 7,5 ; 8,0$ и 8,5. Очаги локализованы в Камчатской зоне субдукции у берегов п-ова Камчатка, приблизительно в районе генерации землетрясения 1952 г. M 9,0. Данные события потенциально способны вызвать волны цунами различной интенсивности. Очаги представлены одной дислокационной плоскостью с равномерным распределением смещения. Длина L и ширина $\mathrm{W}$ плоскости дислокаций и величина смещения в очаге D были рассчитаны по формулам (1)-(4) [7].

$$
\begin{gathered}
M_{w}=\frac{2}{3} \log _{10} M_{0}-10,7, \\
\log _{10} L=-2,28+0,55 \times M_{w}, \\
\log _{10} W=-1,8+0,45 \times M_{w}, \\
M_{0}=\mu L W D .
\end{gathered}
$$

Полученные с использованием $\mu=3,5 \times$ $\times 10^{10} \mathrm{H} /$ м параметры представлены в табл. 1 .

Таблица 1

Параметры очагов гипотетических землетрясений

\begin{tabular}{|c|c|c|c|}
\hline магнитуда & $\mathrm{L}($ км) & $\mathrm{W}($ км) & $\mathrm{D}(\mathrm{M})$ \\
\hline $\mathrm{M}_{\mathrm{w}} 7,5$ & 70,00 & 37,58 & 2,16 \\
\hline $\mathrm{M}_{\mathrm{w}} 8,0$ & 131,82 & 63,20 & 3,85 \\
\hline $\mathrm{M}_{\mathrm{w}} 8,5$ & 248,31 & 105,92 & 6,85 \\
\hline
\end{tabular}

Для всех плоскостей глубина залегания верхней грани равна 5 км, угол падения $15^{\circ}$ и направление простирания сейсморазрыва $215^{\circ}$, что в целом соответствует параметрам землетрясений Камчатской зоны субдукции.

Для расчёта косейсмических смещений использованы три модели Земли: I) однородное полупространство с $\mu=3,5 \cdot 10^{10}$ (программный пакет Coulomb3.0), II) сферическая однородная и III) сферическая слоистая модели (программный пакет Static1D). B качестве сферической слоистой была использована модель Preliminary Reference Earth 
Model (PREM), в то время как в сферической однородной земная кора представлена слоем толщиной 61 км с $\mu=3,5 \cdot 10^{10} \mathrm{H} / \mathrm{M}$, коэффициентом Пуассона $v=0,25$ и плотностью $3300 \mathrm{\kappa г} / \mathrm{M}^{3}$.

Для всех очагов и моделей среды были рассчитаны соответствующие им вертикальные косейсмические смещения в узлах сетки с шагом $0,2^{\circ}$ (рис. 1) и максимальные вертикальные сдвиги морского дна (табл. 2). Разность косейсмических смещений дна моря, рассчитанных с использованием сферической однородной и модели однородного полупространства, показывает влияние сферичности, а разность смещений, полученных с использованием слоистой сферической и однородной сферической моделей, отображает влияние слоистости земной коры.

В соответствии с табл. 2 и рис. 1 учёт слоистости и сферичности Земли выража- ется в получении меньших величин вертикальных смещений. Полученная разница достигает 30 см только при самом сильном рассматриваемом землетрясении, при котором дислокации морского дна составляют порядка 3 м.

Далее, были рассчитаны горизонтальные косейсмические смещения в местах расположения станций постоянных ГНССнаблюдений на Дальнем Востоке России (рис. 2). Как и в случае с вертикальными, наименьшие смещения соответствуют слоистой сферической модели, несколько большей величины - однородной сферической, самые большие - однородному полупространству. Влияние слоистости и сферичности значительнее на горизонтальные смещения, чем на вертикальные. Также визуально виден различный вклад сферичности и слоистости в уменьшение величины смещений в зависимости от магнитуды события.

Таблица 2

Максимальные вертикальные косейсмические смещения, соответствующие рассмотренным гипотетическим землетрясениям и моделям Земли

\begin{tabular}{|c|c|c|c|c|c|c|}
\hline \multirow[t]{2}{*}{ Магнитуда } & \multicolumn{3}{|c|}{ Максимальные вертикальные движения морского дна (см) } & \multirow{2}{*}{$\frac{\text { COM3 }}{\text { OПM3 }}$} & \multirow{2}{*}{$\frac{\text { CCM3 }}{\text { COM3 }}$} & \multirow{2}{*}{$\frac{\mathrm{CCM} 3}{\mathrm{O \Pi M}}$} \\
\hline & $\begin{array}{l}\text { Однородное } \\
\text { полупростран- } \\
\text { ство (ОПМЗ) }\end{array}$ & $\begin{array}{c}\text { Сферическая } \\
\text { однородная модель } \\
\text { Земли (СОМЗ) }\end{array}$ & $\begin{array}{c}\text { Слоистая сфериче- } \\
\text { ская модель Земли } \\
\text { (ССМЗ) }\end{array}$ & & & \\
\hline $\mathrm{M}_{\mathrm{w}} 7,5$ & 87,5 & 85,9 & 77,5 & 0,98 & 0,90 & 0,88 \\
\hline$M_{w} 8,0$ & 171,4 & 162,4 & 148,1 & 0,95 & 0,91 & 0,86 \\
\hline $\mathrm{M}_{\mathrm{w}} 8,5$ & 307,4 & 297,7 & 276,7 & 0,97 & 0,93 & 0,90 \\
\hline
\end{tabular}
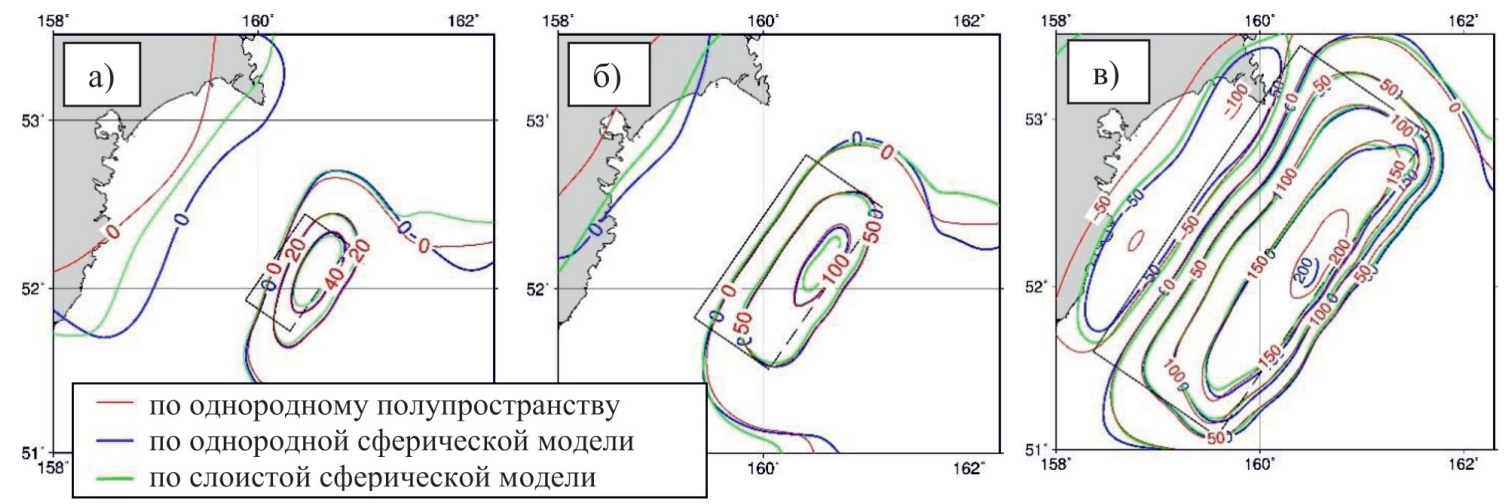

Рис. 1. Вертикальные косейсмические смещения, рассчитанные с использованием различных моделей Земли и магнитуд гипотетических землетрясений: а) $M_{w} 7,5$, б) $M_{w} 8,0$, в) $M_{w} 8,5$. Для $M_{w}$ 7,5 изолинии проведены через 20 см, для $M_{w} 8,0$ и $M_{w} 8,5$ - через 50 см. Красной линией обозначень смещения, рассчитанные с помощью однородного полупространства, синей линией - по однородной сферической модели, зелёной - по слоистой сферической модели. Проекичи плоскостей очагов отображены чёрными прямоугольниками, верхняя грань отображена прерывистой линией 


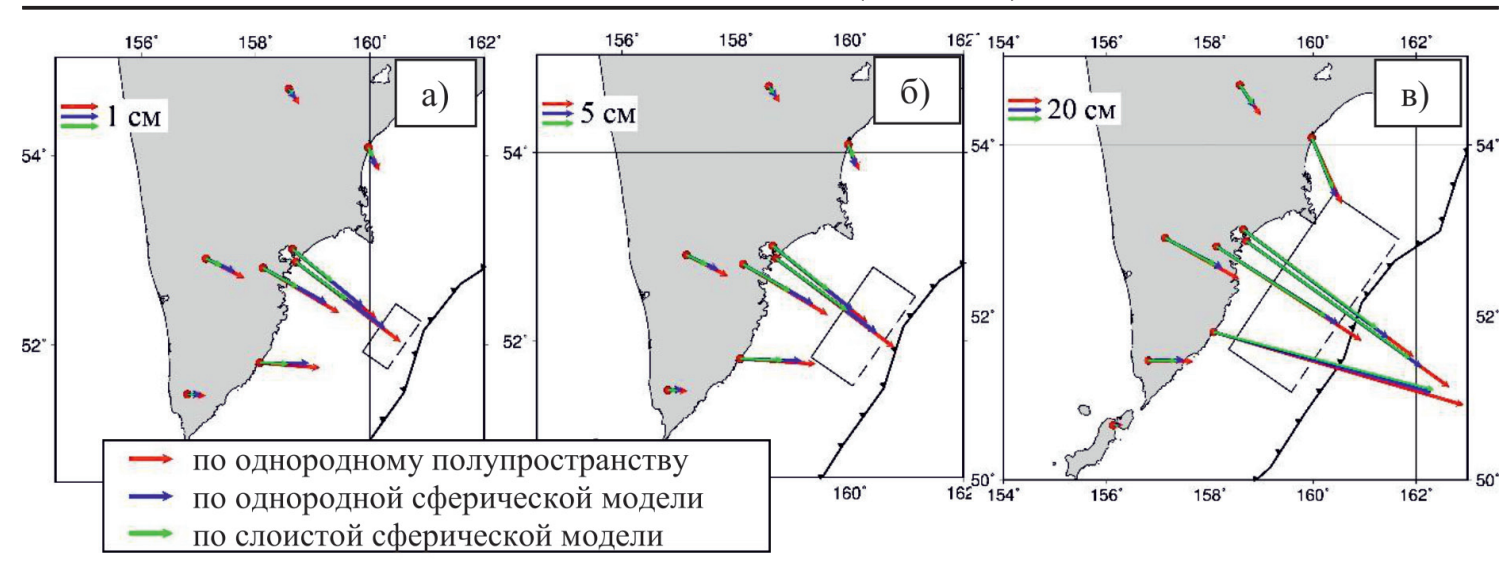

Рис. 2. Горизонтальные косейсмические смещения, рассчитанные на пунктах региональных ГНСС-сетей с использованием моделей-сферической слоистой (зелёные векторы), однородной сферической (синие векторы) и однородного полупространства (красныле векторы). Использовались гипотетические очаги: а) $M_{w} 7,5$, б) $M_{w} 8,0$, в) $M_{w} 8,5$

Так, для очага $\mathrm{M}_{\mathrm{w}}$ 7,5 эффект слоистости земной коры ведёт к более значимому преуменьшению смещений, чем сферичность поверхности. Для $\mathrm{M}_{\mathrm{w}} 8,0$, заметно примерно равное влияние факторов, а при $\mathrm{M}_{\mathrm{w}} 8,5$ слоистость практически не влияет на смещения, и к уменьшению их величин, согласно расчётам, ведёт сферичность поверхности. При этом следует заметить, что по отношению к очагам землетрясений рассматриваемые ГНСС-пункты принадлежат к разным зонам - ближней и дальней, в зависимости от магнитуды события. Для события $\mathrm{M}_{\mathrm{w}}$ 7,5 все ГНСС-станции будут принадлежать дальней зоне, для $\mathrm{M}_{\mathrm{w}} 8,0$ в неё попадают только ближайшие станции, расположенные на побережье Камчатского п-ова, а для землетрясения $\mathrm{M}_{\mathrm{w}} 8,5$ ближняя зона охватывает уже все станции южной части Камчатки.

Таким образом, можно предположить, что чем сильнее событие и ближе наблюдаемые пункты находятся к очагу, тем больший вклад в уменьшение смещений оказывает сферичность поверхности по сравнению со слоистостью. И наоборот, чем слабее очаг и чем дальше от него расположены пункты наблюдения, тем заметнее влияние слоистости земной коры. В любом случае, оба рассматриваемых фактора приводят к уменьшению косейсмических смещений, причём в большей степени этот эффект сказывается на горизонтальной компоненте сдвигов.

\section{Инверсия}

Слоистая сферическая модель намного точнее описывает действительное строение
Земли, нежели однородное полупространство. Поэтому для дальнейших вычислений мы приняли косейсмические смещения, рассчитанные с помощью модели PREM в качестве реальных подвижек. Как было показано выше, использование однородного полупространства в качестве модели Земли при моделировании косейсмических смещений приводит к переоценке их значений. И наоборот, инвертирование реальных смещений без учёта слоистости и сферичности должно привести к недооценке магнитуды землетрясения и, соответственно, цунамиопасности.

Для наглядной демонстрации этого эффекта магнитуды гипотетических землетрясений были переопределены путём инверсии по методу [4] рассчитанных с использованием сферической слоистой модели косейсмических смещений. При инверсии пространственные характеристики очагов были зафиксированы, и определялась только величина смещения в сейсмофокальной плоскости $\left(\mathrm{D}_{\text {inverted }}\right)$. Косейсмическим смещениям присвоены ошибки величиной 1 мм, по аналогии с точностями реальных измерений. После определения $\mathrm{D}_{\text {inverted }}$ по формулам (1)-(4) были определены магнитуды инвертированных событий $\mathrm{M}_{\text {winverted }}$ (табл. 3).

Инвертированные значения смещения в очаге $\mathrm{D}_{\text {inverted }}$ меньше опорных (исходных) $\mathrm{D}_{\text {reference }}$ В абсолютных значениях разница варьируется от 1 м при $\mathrm{M}_{\mathrm{w}} 8,5$ до 1,53 м при $\mathrm{M}_{\mathrm{w}} 8,0$. Однако при относительном сравнении $\mathrm{D}_{\text {reference }}$ и $\mathrm{D}_{\text {inverted }}$ становится очевидным, что расхождение уменьшается с ростом 
магнитуды. При $\mathrm{M}_{\mathrm{w}}$ 7,5 недооценка составляет около $50 \%$, при $\mathrm{M}_{\mathrm{w}} 8,5-15 \%$ от опорных значений. Для большей наглядности были смоделированы поля вертикальных косейсмических смещений (инвертированные поля вертикальных смещений) с использованием инвертированных параметров землетрясений и модели Земли PREM (рис. 3). Максимальные косейсмические смещения согласно опорной disp reference $_{\text {и инвертиро- }}$

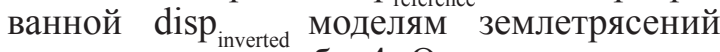
представлены в табл. 4. Относительное соотношение disp max $_{\text {inverted и disp }}$ reference идентично отношениям $\mathrm{D}_{\text {reference }}$ и $\mathrm{D}_{\text {inverted }}$. При этом абсолютное максимальное pacхождение в 60 см наблюдается при $\mathrm{M}_{\mathrm{w}} 8.0$.

С другой стороны, если в СПЦ на всех этапах обработки адаптировано однородное полупространство в качестве модели
Земли, то недооценка дислокации в очаге может быть частично компенсирована переоценкой смещений морского дна в ходе последующего моделирования. В связи с этим предположением также были рассчитаны поля смещений морского дна с использованием инвертированных параметров землетрясений и однородного полупространства как модели Земли (табл. 4, рис. 3). В данном случае, действительно, инвертированные поля имеют меньшее расхождение с опорными. Для $\mathrm{M}_{\mathrm{w}} 8,0$ и $\mathrm{M}_{\mathrm{w}} 8,5$ улучшение составило $10 \%$ от опорных значений по сравнению с применением слоистой сферической модели. Максимальная недооценка наблюдается при $\mathrm{M}_{\mathrm{w}} 8,0$ и равна 45 см. Для события $\mathrm{M}_{\mathrm{w}} 8,5$ недооценка минимальна как в абсолютном (14 см), так и в относительном (5\%) отношениях.

Таблица 3

Опорные и инвертированные значения магнитуд землетрясений и смещения в очаге

\begin{tabular}{|c|c|c|c|c|}
\hline магнитуда & $\mathrm{D}_{\text {reference }}(\mathrm{cm})$ & $\mathrm{D}_{\text {inverted }}(\mathrm{cm})$ & $\mathrm{D}_{\text {inverted }} \mathrm{D}_{\text {reference }}$ & $\mathrm{M}_{\text {winverted }}$ \\
\hline $\mathrm{M}_{\mathrm{w}} 7,5$ & 216,0 & 105,5 & 0,49 & 7,29 \\
\hline $\mathrm{M}_{\mathrm{w}} 8,0$ & 385,0 & 231,4 & 0,60 & 7,85 \\
\hline $\mathrm{M}_{\mathrm{w}} 8,5$ & 685,4 & 585,4 & 0,85 & 8,49 \\
\hline
\end{tabular}

Таблица 4

Опорные и инвертированные максимальные вертикальные смещения морского дна

\begin{tabular}{|c|c|c|c|c|}
\hline \multirow[t]{2}{*}{ Магнитуда } & \multicolumn{3}{|c|}{ Максимальные вертикальные движения морского дна (см) } & \multirow{2}{*}{$\begin{array}{l}\text { disp }_{\text {inverted }} \text { (однородное полу- } \\
\text { пространство)/disp } \\
\text { reference }\end{array}$} \\
\hline & disp $_{\text {reference }}$ & $\operatorname{disp}_{\text {inverted }}(\mathrm{PREM})$ & $\begin{array}{c}\text { disp }_{\text {inverted }} \text { (однородное } \\
\text { полупространство) }\end{array}$ & \\
\hline $\mathrm{M}_{\mathrm{w}} 7,5$ & 77,5 & 37,9 & 42,7 & 0,55 \\
\hline $\mathrm{M}_{\mathrm{w}} 8,0$ & 148,1 & 89,0 & 103,0 & 0,70 \\
\hline $\mathrm{M}_{\mathrm{w}} 8,5$ & 276,7 & 236,4 & 262,5 & 0,95 \\
\hline
\end{tabular}

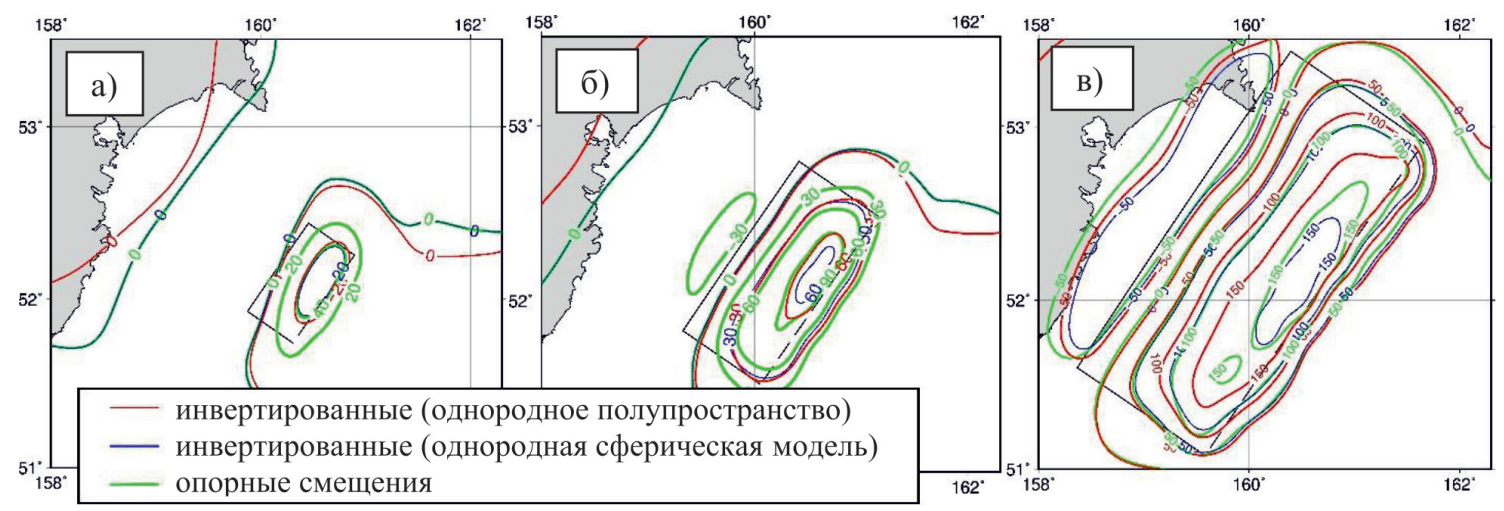

Рис. 3. Вертикальные поля смещений, соответствующие опорным (зелёные изолинии) и инвертированным очагам (красные изолинии - смещения рассчитаны в однородном полупространстве, синие изолинии - с использованием слоистой сферической модели Земли). Магнитуды гипотетических землетрясений: а) $M_{w} 7,5$, б) $M_{w} 8,0$, в) $M_{w} 8,5$ 
Для проверки применённой методологии, косейсмические смещения, рассчитанные в однородном полупространстве на ГНСС-станциях, были инвертированы, и полученные магнитуды достигли значений исходных, что говорит о корректности применяемого подхода.

\section{Заключение}

На примере трёх гипотетических землетрясений различной мощности было рассмотрено влияние используемой модели Земли на величины моделируемых косейсмических смещений, а также на результаты инверсии. Показано, что модель Земли существенным образом влияет на величины смещений, причём данное влияние более значимо по горизонтальной компоненте. Кроме того, учёт сферичности и слоистости влияет на смоделированные величины смещений неравномерно и варьируется в зависимости от силы землетрясения и дальности расположения наблюдаемых пунктов от очага. Тем не менее некоторые закономерности очевидны: влияние слоистости коры усиливается при удалении от очага, а сферичности поверхности - при увеличении магнитуды землетрясения и приближении к нему. Игнорирование обоих факторов ведёт к переоценке величин смещений в ходе моделирования.

Для целей СПЦ важно хорошее соответствие реальных смещений морского дна (в нашем случае - опорных смещений) с инвертированными. При использовании однородного полупространства, несмотря на некоторую недооценку величины дислокации в очаге и, соответственно, магнитуды землетрясения, недооценка инвертированных смещений в худшем случае не превышает 50 см. Наилучшее соответствие получено при наиболее сильном событии $\mathrm{M}_{\mathrm{w}} 8,5$.

Если учесть, что одноплоскостная модель землетрясения не способна точным образом описать реально возникшие смещения в очаге землетрясения, то полученные расхождения можно считать приемлемыми, по крайней мере для наиболее сильных землетрясений. В случае событий $\mathrm{M}_{\mathrm{w}} \leq 8,0$ недооценка максимальных смещений морского дна достигает половины от опорных значений, что может отразиться на выявлении самого факта цунами при его моделировании.
Исходя из вышесказанного, можно заключить, что в случае сильных землетрясений $\mathrm{M}_{\mathrm{w}} \approx 8,5$, происходящих в зоне субдукции на небольшом удалении от побережья и ГНСС-станций, пренебрежение слоистостью и сферичностью не является критическим. С другой стороны, для событий $\mathrm{M}_{\mathrm{w}} \leq 8,0$ всё же желательно использование более точной модели Земли.

Таким образом, при возникновении сильных землетрясений задействование наиболее простой модели Земли (однородное полупространство) в СПЦ способствует довольно точному и наиболее быстрому на данный момент определению начальных параметров волны цунами.

Работа выполнена при поддержке Японско-Российского ичентра молодёжных обменов, а также Института прикладной математики Дальневосточного отделения Российской академии наук.

\section{Список литературы / References}

1. Kawamoto S., Ohta Y., Hiyama Y., Todoriki M., Nishimura T., Furuya T., Sato Y., Yahagi T., Miyagawa K. REGARD: A new GNSS-based real-time finite fault modeling system for GEONET. Journal of Geophysical Research: Solid Earth, 2017 February. Vol. 122. 26 p. DOI: 10.1002/2016JB013485.

2. Melgar D., Geng J., Crowell B.W., Haase J.S., Bock Y., Hammond W.C., Allen R.M. Seismogeodesy of the $2014 \mathrm{M}_{\mathrm{w}} 6.1$ Napa earthquake, California: Rapid response and modeling of fast rupture on a dipping strike-disp fault. Journal of Geophysical Research: Solid Earth. 2015 May. Vol. 120. 22 p. DOI: $10.1002 / 2015 J B 011921$.

3. Crowell B.W., Schmidt D.A., Bodin P., Vidale J.E., Gomberg J., Hartog J.R., Kress V.C., Melbourne T.I., Santillan M., Minson S.E., Jamison D.G.. Demonstration of the Cascadia G-FAST Geodetic Earthquake Early Warning System for the Nisqually, Washington, Earthquake. Seismological Research Letters. 2016. Vol. 87. Number 4 July/August. 14 p. DOI: 10.1785/0220150255.

4. Matsuura M., Hasegawa Y. A maximum likelihood approach to nonlinear inversion under constraints. Physics of the Earth and planetary interiors. 1987. Vol. 47. P. 179-187.

5. Banerjee P., Pollitz F.F., Bürgmann R. The Size and Duration of the Sumatra-Andaman Earthquake from Far-Field Static Offsets. Science. 2005. Vol. 308. Issue 5729. P. 1769-1772. DOI: $10.1126 /$ science. 1113746.

6. Нечаев Г.В., Шестаков Н.В., Такахаши Х., Герасименко М.Д., Сысоев Д.В. О применении методов Глобальных навигационных спутниковых систем для целей раннего предупреждения о цунами в Курило-Камчатском регионе // Успехи современного естествознания. 2020. № 3. C. 92-98. DOI: 10.17513/use.37351.

Nechaev G.V., Shestakov N.V., Takahashi H., Gerasimenko M.D., Sysoev D.V. On application of methods of Global Navigation Satellite Systems for tsunami early warning in the Kuril-Kamchatka region // Advances in current natural sciences. 2020. № 3. P. 92-98 (in Russian).

7. Blaser L., Krüger F., Ohrnberger M., Scherbaum F. Scaling Relations of Earthquake Source Parameter Estimates with Special Focus on Subduction Environment. Bulletin of the Seismological Society of America. 2010. Vol. 100. № 6. P. 2914 2926. DOI: $10.1785 / 0120100111$. 